\title{
Model-plant Mismatch Detection of Nonlinear Processes Based on Multi-model LPV Model
}

\author{
Jiangyin Huang ${ }^{1, *}$ and Jing Zhao ${ }^{1}$ \\ ${ }^{1}$ Department of Electrical Engineering and Automation, Xiamen University of Technology, 361024 Xiamen, Fujian, China
}

\begin{abstract}
The performance of model-based controller depends on the quality of the identified model. Accurate detection of the channel with model-plant mismatch can avoid re-identification of the entire multivariable system, thereby reducing the disturbance to normal production caused by identification test. A model-plant mismatch detection methodology for nonlinear systems based on LPV (Linear Parameter Varying) model was proposed in this work. The detection was performed only when the control performace becomes worse. Firstly, the LPV model based on multi-model interpolation was adopted to represent the nonlinear process. Then partial correlation coefficients between the model residuals and the inputs of the models at each of the operation points were analyzed to diagnose the model-plant mismatch of the local models. Finally, the LPV model was re-identified by updating the local mismatch models and re-estimating the model weighing parameters. The experimental results show that the partial correlation coefficient of the mismatch model is obviously larger than that of the exact model, which can point out the channel with model-plant mismatch correctly. The proposed method is suitable for the nonlinear processes which have relative steady states in their operating trajectorys.
\end{abstract}

\section{Introduction}

With the development of automatic control technology, the control algorithm based on process model, such as MPC (Model Predictive Control), has been widely used in industrial system control. The performance of such model based controller depends on the quality of the model which is identified and used in the controller. As the process model may change with the aging of components and the change of operating environment, the control performance will deteriorate as the modelplant mismatch increases. Although new models can be obtained by re-identification, however, for most complex industrial systems, identifying the entire large MIMO models is too costly, and may disturb the normal production because of the identification test signal. In order to solve this problem, in recent years, scholars have begun to study the methods which can be used to diagnose the exact channel with model-plant mismatch. Badwe et al. proposed a method which detects channels with significant mismatch using partial correlations between the model residuals and inputs [1]. It was followed by Loeff who improved this method by considering the influence of system delay [2]. Ji et al. use non-disturbing small sinusoidal test signals to obtain accurate estimated of process frequency response and then detect the model-plant mismatch by comparing the difference between the frequency response of the plant and the MPC model [3]. Two years later, a data driven methodology based on the statistical bands of Markov parameters are proposed [4]. In this method, the model mismatch is detected by evaluating the bias between the band of the normal case and that of the monitored case. Furthermore, the plant-model-ratio is adopted to identify the source of mismatch, namely gain, dynamics and delay mismatches from system closed-loop operation data [5]. A methodology based on a process model evaluation index is proposed in [6], the index is the ratio between the variance of the disturbance innovation and that of the model quality variable. All the detection methods mentioned above can only be used in linear system. However, most of the industrial processes are inherently nonlinear and linear models representing these processes are valid only over a limited range around the operating point. Because the process model will change as the operating point changes, model-plant mismatch detection should be performed according to the process running state, the control parameters are also need to be reset. There are only a few researchers who studied the model-plant mismatch detection of nonlinear systems. Chen et al. proposed a mismatch detection methodology based on mutual information [7]. The effectiveness of the proposed method is only verified on the system with nonlinear input signals. However, for the nonlinear system with changing models along its operation trajectory, the validity of the proposed method is not demonstrated.

In this work, a model-plant mismatch detection methodology for nonlinear systems is proposed. The 
LPV model based on multi-model interpolation is used to represent the nonlinear system first, and then the model-plant mismatch is diagnosed by analyzing the partial correlation coefficients between the model residuals and the system inputs of the local linear models at each of the operating points.

The paper is organized as follows: in Section 2, the problem of model-plant mismatch detection of nonlinear process is discussed and a closed-loop detection methodology based on partial correlation calculation for multi-model based LPV model is proposed. In Section 3, the validity of the proposed methodology is verified by simulating a LPV model. Section 4 gives concluding remarks and future directions.

\section{Model-plant mismatch of LPV model}

\subsection{Problem definition}

Consider a MIMO nonlinear system with $m$ manipulate variables (MVs), $n$ controlled variables (CVs) and $l$ measureable disturbance variables (DVs). Suppose $\mathbf{G}_{u}$ and $\hat{\mathbf{G}}_{u}$ are the $n \times m$ plant and model between the MVs and CVs respectively, $\mathbf{G}_{d}$ and $\hat{\mathbf{G}}_{d}$ are the $n \times l$ plant and model between the DVs and CVs. Consider the closed-loop internal model control (IMC) structure of Fig.1, where $\mathbf{r}(k)$ is the vector of set points, $\mathbf{d}(k)$ is the vector of DVs, $\mathbf{y}(k)$ and $\hat{\mathbf{y}}(k)$ are the plant and model output vectors respectively, $\mathbf{e}(k)=\mathbf{y}(k)-\hat{\mathbf{y}}(k)$ is the vector of model residuals and $\mathbf{v}(k)$ is the vector of Gaussian disturbances acting on the process. $\mathbf{Q}$ is the multivariable controller and $\mathbf{u}(k)$ is the vector of MVs.

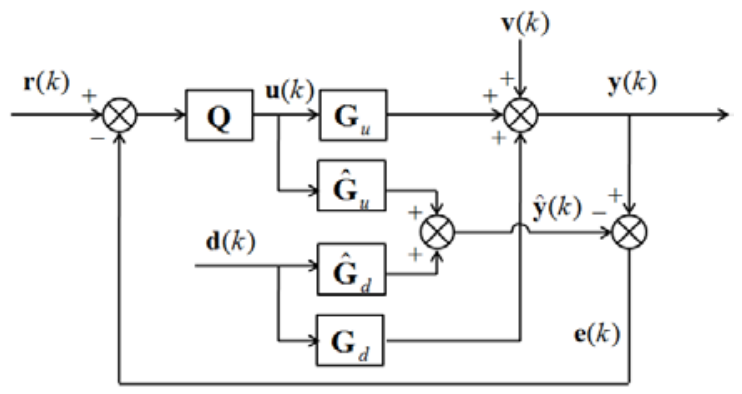

Fig. 1. Closed-loop system: IMC structure

Let $\boldsymbol{\Delta}_{1}=\mathbf{G}_{u}-\hat{\mathbf{G}}_{u}$ be the MV-CV model-plant mismatch, $\Delta_{2}=\mathbf{G}_{d}-\hat{\mathbf{G}}_{d}$ be the DV-CV model-plant mismatch. The main objective of this work is to locate the channel in $\boldsymbol{\Delta}_{1}$ and $\boldsymbol{\Delta}_{2}$ which exists significant model-plant mismatch from the closed-loop data of the process. From Fig.1, the following expressions can be written:

$$
\begin{aligned}
& \mathbf{e}(k)=\Delta_{1} \mathbf{u}(k)+\boldsymbol{\Delta}_{2} \mathbf{d}(k)+\mathbf{v}(k) \\
& =\left[\mathbf{I}+\Delta_{1} \mathbf{Q}\right]^{-1} \Delta_{1} \mathbf{Q} \mathbf{r}(k)+\left[\mathbf{I}+\Delta_{1} \mathbf{Q}\right]^{-1} \Delta_{2} \mathbf{d}(k)+\left[\mathbf{I}+\Delta_{1} \mathbf{Q}\right]^{-1} \mathbf{v}(k) \\
& \mathbf{u}(k)=\mathbf{Q}[\mathbf{r}(k)-\mathbf{e}(k)] \\
& =\mathbf{Q}\left[\mathbf{I}+\boldsymbol{\Delta}_{1} \mathbf{Q}\right]^{-1} \mathbf{r}(k)-\mathbf{Q}\left[\mathbf{I}+\boldsymbol{\Delta}_{1} \mathbf{Q}\right]^{-1} \boldsymbol{\Delta}_{2} \mathbf{d}(k)-\mathbf{Q}\left[\mathbf{I}+\boldsymbol{\Delta}_{1} \mathbf{Q}\right]^{-1} \mathbf{v}(k)
\end{aligned}
$$

According to the first line of Eq. (1), $\Delta_{1}$ and $\Delta_{2}$ can be detected by analyzing the correlations between $\mathbf{u}(k)$ and $\mathbf{e}(k)$ and the correlations between $\mathbf{d}(k)$ and $\mathbf{e}(k)$. However, there are three problems which should be solved in the analysis of such correlations.

(1)The disturbance vector $\mathbf{v}(k)$ is immeasurable in industrial process and is associated with model residuals. The correlations between $\mathbf{v}(k)$ and $\mathbf{e}(k)$ will influence the correlations between $\mathbf{e}(k)$ and the MVs/DVs. Therefore, these effects of $\mathbf{v}(k)$ on $\mathbf{e}(k)$ must be removed.

(2)There maybe correlations amongst the MVs and the DVs themselves because of the feedback loop and the controller. Such correlations will also confound the regular correlation analysis between $\mathbf{e}(k)$ and the MVs/DVs. Therefore, all of these correlations should also be decoupled.

(3)The plants $\mathbf{G}_{u}$ and $\mathbf{G}_{d}$ are both nonlinear, however, in order to reduce control difficulty, linear models are often used in internal model controller, namely, the models $\hat{\mathbf{G}}_{u}$ and $\hat{\mathbf{G}}_{d}$ are both linear. Therefore, the characteristics of $\Delta_{1}$ and $\Delta_{2}$ may be nonlinear and time-varying, which significantly increase the difficulty of analyzing the above correlations.

In order to solve the first two problems, an improved partial correlation analysis is adopted to remove the spurious correlations in this work. The traditional partial correlation analysis is often used to detect linear relations between some variables, especially when the variables are correlated amongst themselves. Abhijit et. al.[1] has adopted that method in the detection of modelplant mismatch for linear systems. However, if the plant is nonlinear and the model is linear, the relations between $\mathbf{e}(k)$ and $\mathbf{u}(k)$ and the relations between $\mathbf{e}(k)$ and $\mathbf{d}(k)$ are both nonlinear. In order to make the methodology of partial correlation analysis effective, LPV model based on multi model interpolation is used to represent the nonlinear plant.

\subsection{Model-plant mismatch detection of LPV model}

\subsubsection{Multi-model based LPV model}

Zhu and Xu [8] proposed a LPV model structure based on the interpolation of local linear models. An important assumption for such LPV model is that, although most industrial systems are nonlinear, their trajectories are composed of some steady states (operation points) and transitional stages. In the steady states, the plant is regarded as linear and is represented by a linear model, and then the models of the transitional stages are obtained by interpolating these local linear models. Consider the nonlinear MIMO process discussed in Section 2.1, without loss of generality, assume that there are 3 steady states on the operating trajectory of this 
process, $\hat{\mathbf{y}}^{i}(k)$ is the output vector of the identified linear model of the $i^{\text {th }}$ steady state:

$$
\hat{\mathbf{y}}^{i}(k)=\hat{\mathbf{G}}_{u}^{i} \mathbf{u}(k)+\hat{\mathbf{G}}_{d}^{i} \mathbf{d}(k),(i=1,2,3)
$$

where $\hat{\mathbf{G}}_{u}^{i}$ and $\hat{\mathbf{G}}_{d}^{i}$ are the MV-CV and DV-CV linear model of the $i^{\text {th }}$ steady state. Then the output vector of LPV model $\hat{\mathbf{y}}(k)$ is obtained by interpolating the linear models in (3):

$$
\hat{\mathbf{y}}(k)=\sum_{i=1}^{3} \alpha^{i}(w(k)) \hat{\mathbf{y}}^{i}(k)
$$

where $\alpha^{i}(w(k))$ are the model weights which are static functions of the scheduling variable $w(k)$ and satisfy $\sum_{i=1}^{3} \alpha^{i}(w(k))=1$. The scheduling variable is a measurable or a computable variable which can reflect the operating trajectory of the process. Namely, in the steady states, the value of the scheduling variable is constant. In extreme cases, when the process runs in a steady state, the weight of the corresponding linear model will reach its maximum value of 1 and the weights of all the other models are 0. It can be seen from Eq.(4) that the LPV model at each steady state is linear because the model weights are constant, therefore, the methodology of partial correlation analysis can be used to analyze the linear relations between the model residuals and $\mathrm{MVs} / \mathrm{DV}$ s at the steady states.

\subsection{2 model-plant mismatch detection using partial correlation analysis}

There are two kinds of errors that could lead to modelplant mismatch of LPV model. One is the model-plant mismatch of the local linear models, the other one is the inaccurate parameters of model weights. In the identification of LPV model, the identification experiments are usually carried out in each steady state first to identify the local linear models. Then, the weighting parameters are estimated by minimizing the model output error. The accuracy of the local linear models not only affects the estimation of weighting parameters, but also affects the accuracy of the LPV model. Therefore, the detection of model-plant mismatch of LPV model should also involves two steps. First, the quality of the local models is detected; secondly, if there are mismatch local models, the weighting parameters should be re-estimated.

Abhijit et. al.[1]pointed out that in the absence of setpoint $\mathbf{r}(k)$ changes, it would not be possible to determine the extent of mismatch because of the direct causal relation between $\mathbf{e}(k)$ and $\mathbf{u}(k)$. Therefore, when the mismatch diagnosis is performed, additional excitation signal should be superimposed on $\mathbf{r}(k)$, which will cause interference to the normal production. In order to minimize such disturbance the model mismatch diagnosis is started only when the control performance become worse. As we know, the performance of modelbased control system depends a lot on the process model quality. Although the decrease in control quality does not always come from model-plant mismatch, e.g., it may also caused by inaccurate control parameters, defective valve or significant disturbance, the control error of the process can still be used as the criterion for the model mismatch detection. Considering the influence of the historical control error, define the integrated control error $J$ of the process in (5):

$$
\begin{gathered}
J(k)=\sum_{i=1}^{n} \lambda_{i} J_{i}(k) ; \\
J_{i}(k)=\gamma e_{c i}^{2}(k)+\eta \sum_{j=1}^{L} \rho_{j} e_{c i}^{2}(k-j),(i=1,2, \ldots, n)
\end{gathered}
$$

where $J_{i}(k)$ is the cumulative control error [9] of the $i^{\text {th }}$ output in finite time domain $L, \lambda_{i}$ is the weight of $J_{i}(k)$ and satisfy $\sum_{i=1}^{n} \lambda_{i}=1 . e_{c i}(k)$ is the control error of the $i^{\text {th }}$ output at sampling time $k$, it depends on the difference between the setting value and the actual value of the corresponding output. $\gamma$ and $\eta$ are the weights of the current and historical control error respectively, $\rho_{j}$ is the forgetting factor of the historical control error and satisfy $\sum_{j=1}^{L} \rho_{j}=1$. All the weights $\lambda_{i}, \gamma, \eta, \rho_{j}$ can be set according to the prior knowledge of the process.

When $J(k)$ is greater than the specified threshold, it is reasonable to assume that the control performance is not good enough and model-plant mismatch of the LPV model need to be diagnosed.

According to the previous analysis, the mismatch of the local linear models of each of the steady states is diagnosed first. An improved partial correlation analysis is developed and the various steps in the proposed methodology are as follows:

Step1: Collect the process closed-loop data, including the setpoints, MVs, DVs, CVs and model residuals from each of the steady states. Make sure that the setpoints are sufficiently exciting, if not, continuous excitation signal, e.g. GBN (Generalized Binary Noise) signal need to be superimposed on the set point to ensure the estimation accuracy of the models which will be identified in Step2.

Step2: In order to reduce the influence of unmeasurable disturbance on the mismatch detection, the models between MVs and setpoints and the models between MVs and DVs are identified and then the disturbance free components of the MVs are found. Please note that the data of each steady state should be handled by the same way. The processing process of the data of each steady state is described as follows.

Let $\mathbf{Q}\left[\mathbf{I}+\Delta_{1} \mathbf{Q}\right]^{-1}=\mathbf{S}_{r u}$ and $\mathbf{Q}\left[\mathbf{I}+\Delta_{1} \mathbf{Q}\right]^{-1} \boldsymbol{\Delta}_{2}=\mathbf{S}_{d u}$, the expression in Eq.(2) can be written as

$$
\begin{aligned}
\mathbf{u}(k) & =\mathbf{S}_{r u} \mathbf{r}(k)-\mathbf{S}_{d u} \mathbf{d}(k)-\mathbf{S}_{r u} \mathbf{v}(k) \\
& =\mathbf{u}^{r}(k)+\mathbf{u}^{d}(k)+\mathbf{u}^{v}(k)
\end{aligned}
$$


where $\mathbf{u}(k)$ is the $\mathrm{MV}$ data of the steady states, $\mathbf{u}^{r}(k), \mathbf{u}^{d}(k)$, and $\mathbf{u}^{v}(k)$ are the components of $\mathbf{u}(k)$ which contain effects of the setpoints, measurable disturbances and unmeasurable disturbances respectively. Denote $\hat{\mathbf{S}}_{r u}$ and $\hat{\mathbf{S}}_{d u}$ are the identified model matrix of $\mathbf{S}_{r u}$ and $\mathbf{S}_{d u}$, then $\mathbf{u}^{r}(k), \mathbf{u}^{d}(k)$ can be approximated from (7).

$$
\hat{\mathbf{u}}^{r}(k)=\hat{\mathbf{S}}_{r u} \mathbf{r}(k) ; \quad \hat{\mathbf{u}}^{d}(k)=-\hat{\mathbf{S}}_{d u} \mathbf{d}(k)
$$

$\hat{\mathbf{u}}^{r}(k)$ and $\hat{\mathbf{u}}^{d}(k)$ are both free from $\mathbf{v}(k)$ because $\mathbf{r}, \mathbf{d}$ and $\mathbf{v}$ are uncorrelated to each other. According to the analysis in section 2.1, the model-plant mismatch $\boldsymbol{\Delta}_{1}$ and $\boldsymbol{\Delta}_{2}$ can be detected by analyzing the correlation between $\mathbf{e}(k)$ and $\hat{\mathbf{u}}^{r}(k)$ and the correlation between $\mathbf{e}(k)$ and $\hat{\mathbf{u}}^{d}(k)$. Because there are $m$ MVs, $n$ $\mathrm{CVs}$ and $l \mathrm{DVs}$ of the process, the components of $\mathbf{e}(k) \quad, \quad \hat{\mathbf{u}}^{r}(k) \quad$ and $\quad \hat{\mathbf{u}}^{d}(k) \quad$ can be written as $\mathbf{e}(k)=\left[e_{1}(k) e_{2}(k) \cdots e_{n}(k)\right], \hat{\mathbf{u}}^{r}(k)=\left[\hat{u}_{1}^{r}(k) \hat{u}_{2}^{r}(k) \cdots \hat{u}_{m}^{r}(k)\right]$, $\hat{\mathbf{u}}^{d}(k)=\left[\hat{u}_{1}^{d}(k) \hat{u}_{2}^{d}(k) \cdots \hat{u}_{l}^{d}(k)\right]$.

Step3: Diagnose the mismatch in $\Delta_{1}$ of the local linear models at each of the steady states in turn by calculating the partial correlations between each item of $\mathbf{e}(k)$ and each item of $\hat{\mathbf{u}}^{r}(k)$. Take the channel $u_{j}-y_{i}$ as an example ( $u_{j}$ is the $j^{\text {th }} \mathrm{MV}, y_{i}$ is the $i^{\text {th }} \mathrm{CV}$ ), the calculation process of the partial correlation is:

Firstly, find the component of $\hat{u}_{j}^{r}(k)$ that is uncorrelated with other MVs and the DVs. Assume the relations between $\hat{u}_{j}^{r}(k)$ and other MVs and the relations between $\hat{u}_{j}^{r}(k)$ and the DVs can be expressed as (8):

$$
\hat{u}_{j}^{r}(k)=\widetilde{\mathbf{u}}^{r}(k) \boldsymbol{\theta}_{u_{j}}^{r}+\hat{\mathbf{u}}^{d}(k) \boldsymbol{\theta}_{u_{j}}^{d}+\varepsilon_{u_{j}}(k)
$$

where $\widetilde{\mathbf{u}}^{r}(k)$ contains all the component of $\hat{\mathbf{u}}^{r}(k)$ except $\hat{u}_{j}^{r}(k), \quad \boldsymbol{\theta}_{u_{j}}^{r}$ and $\boldsymbol{\theta}_{u_{j}}^{d}$ are the parameter matrices which can be identified by Least-squares method from the experimental data. $\varepsilon_{u_{j}}(k)$ is the component of $\hat{u}_{j}^{r}$ that is free of effects from the other MVs as well as all DVs, the estimate of $\varepsilon_{u_{j}}(k)$ can be obtained as

$$
\hat{\varepsilon}_{u_{j}}(k)=\hat{u}_{j}^{r}(k)-\widetilde{\mathbf{u}}^{r}(k) \hat{\boldsymbol{\theta}}_{u_{j}}^{r}-\hat{\mathbf{u}}^{d}(k) \hat{\boldsymbol{\theta}}_{u_{j}}^{d}
$$

Similarly, find the component of $e_{i}(k)$ that is uncorrelated with all the MVs expect $\hat{u}_{j}^{r}(k)$ and the DVs:

$$
e_{i}(k)=\widetilde{\mathbf{u}}^{r}(k) \boldsymbol{\theta}_{e_{i}}^{r}+\hat{\mathbf{u}}^{d}(k) \boldsymbol{\theta}_{e_{i}}^{d}+\varepsilon_{e_{i}}(k)
$$

and obtain the estimate of $\varepsilon_{e_{i}}(k)$ :

$$
\hat{\varepsilon}_{e_{i}}(k)=e_{i}(k)-\widetilde{\mathbf{u}}^{r}(k) \hat{\boldsymbol{\theta}}_{e_{i}}^{r}-\hat{\mathbf{u}}^{d}(k) \hat{\boldsymbol{\theta}}_{e_{i}}^{d}
$$

The correlation between $\hat{\varepsilon}_{u_{j}}(k)$ and $\hat{\varepsilon}_{e_{i}}(k)$ indicates the extent of model-plant mismatch in the $u_{j}-y_{i}$ channel.
Then the model-plant mismatch in the $u_{j}-y_{i}$ channel is diagnosed by the cross correlation coefficient between $\hat{\boldsymbol{\varepsilon}}_{u_{j}}$ and $\hat{\boldsymbol{\varepsilon}}_{e_{i}}$. In order to make the diagnosis result more reliable, the cross correlation coefficient in (12) between the components of $\hat{\boldsymbol{\varepsilon}}_{u_{j}}$ and $\hat{\boldsymbol{\varepsilon}}_{e_{i}}$ with the interval of $D$ is considered in turn:

$$
R_{\varepsilon_{\varepsilon_{i}-\varepsilon_{u_{j}}}}(k)=\frac{C_{\varepsilon_{\varepsilon_{i}} \varepsilon_{u_{j}}}(k)}{\sigma_{e_{i}} \sigma_{u_{j}}}
$$

where $C_{\varepsilon_{\varepsilon_{i}} \varepsilon_{u_{j}}}(k)$ is the cross-covariance between $\varepsilon_{e_{i}}(k)$ and $\varepsilon_{u_{j}}(k)$ and satisfy

$$
C_{\varepsilon_{\varepsilon_{i}-} \varepsilon_{u_{j}}}(k)=\frac{1}{N-D} \sum_{t=1}^{N-D}\left[\hat{\varepsilon}_{e_{i}}(t+k)-\bar{\varepsilon}_{e_{i}}\right]\left[\hat{\varepsilon}_{u_{j}}(t)-\bar{\varepsilon}_{u_{j}}\right]
$$

$\sigma_{e_{i}}$ and $\sigma_{u_{j}}$ are the standard deviations of $\hat{\boldsymbol{\varepsilon}}_{u_{j}}$ and $\hat{\boldsymbol{\varepsilon}}_{e_{i}}$ :

$$
\sigma_{e_{i}}=\sqrt{\sum_{t=1}^{N-D}\left(\hat{\varepsilon}_{e_{i}}(t+k)-\bar{\varepsilon}_{e_{i}}\right)^{2}} ; \quad \sigma_{u_{j}}=\sqrt{\sum_{t=1}^{N-D}\left(\hat{\varepsilon}_{u_{j}}(t)-\bar{\varepsilon}_{u_{j}}\right)^{2}}
$$

According to the concept of correlation coefficient, the range of $R_{\varepsilon_{\varepsilon_{i}}-\varepsilon_{u_{j}}}(k)$ is $[-1,1]$, if $R_{\varepsilon_{\varepsilon_{i}}-\varepsilon_{u_{j}}}(k) \neq 0$, it can be considered that there exits model-plant mismatch in $u_{j}-y_{i}$ channel.

Step4: Diagnose the mismatch in $\Delta_{2}$ of the local linear models at each of the steady states in turn by calculating the partial correlations between each item of $\mathbf{e}(k)$ and each item of $\hat{\mathbf{u}}^{d}(k)$. The calculation process is mostly the same as the process described in Sep3, so we won't go into much detail here.

All the mismatch local linear models should be reidentified after they are found. It is worth noting that the identification test signals are only superimposed on the inputs corresponding to the mismatch models. Since the whole MIMO process is not needed to be identified, the inference to the normal production of the process is greatly reduced. When all the mismatch local models are corrected, the new LPV model can be obtained by reestimating the weighting parameters in Eq.(4) according to the weighting function it used. The detailed estimation method of LPV model with linear weight, polynomial weight and Gaussian weight is discussed in [10].

\section{Simulation case studies}

A MIMO nonlinear system with two MVs, two CVs and one DV is considered as our case study. For simulating the discrete process, a sampling period of $1 \mathrm{~min}$ is used. The system is controlled by a multiple MPC controller module in MATLAB. The control parameters are adjusted automatically as the operation status changes. The plant is represented as (15):

$$
\mathbf{y}=\left[\begin{array}{ll}
\frac{2 w}{\left(w^{2}+20\right) s+1} e^{-5 s} & \frac{2 w^{2}}{(15 w+12) s+1} e^{-4 s} \\
\frac{1.6 w+2}{\left(4 w^{2}+10\right) s+1} e^{-6 s} & \frac{w^{3}+2}{\left(5 w^{2}+25\right) s+1} e^{-3 s}
\end{array}\right] \mathbf{u}+\left[\begin{array}{l}
\frac{0.5}{1.85 s+1} e^{-s} \\
\frac{0.25}{2.65 s+1} e^{-s}
\end{array}\right] d
$$


where $w$ is the scheduling variable which changes as the system's operation status changes. Assume there are three steady states and two transient processes along the operating trajectory of the plant. A closed-loop simulation test lasted for 5000 sampling points is performed and the trajectory of $w$ is shown in Fig.2. The setting values of the CVs are GBN sequences. In order to simulate unmeasured process disturbance, filtered white noise sequences are added at the two CVs.

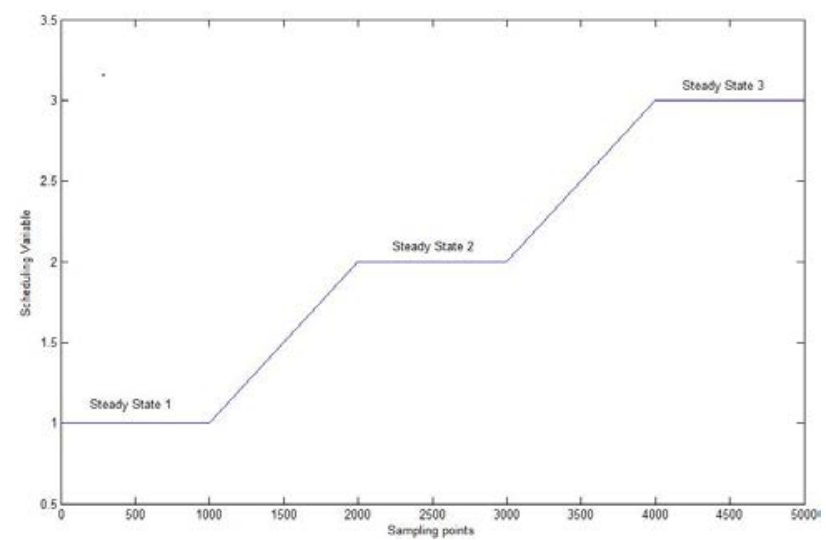

Fig. 2. The trajectory of the scheduling variable

Assume that there is no model-plant mismatch at the second steady state $(w=2)$ and some identified models which are used in MPC at the other steady states are not accurate. The differences between the plant and the model are listed in Table 1. The models which are not listed in the table are the same as the plant.

Table 1. Set of model-plant mismatch

\begin{tabular}{|c|c|c|c|}
\hline $\begin{array}{c}\text { Scheduling } \\
\text { variable }\end{array}$ & Channel & Plant & Model \\
\hline \multirow{2}{*}{$w=1$} & $G_{12}$ & $\frac{2}{27 s+1} e^{-4 s}$ & $\frac{4}{27 s+1} e^{-4 s}$ \\
\hline \multirow{2}{*}{$w=3$} & $G_{21}$ & $\frac{6.8}{46 s+1} e^{-6 s}$ & $\frac{8}{35 s+1} e^{-5 s}$ \\
\cline { 2 - 4 } & $G_{22}$ & $\frac{29}{70 s+1} e^{-3 s}$ & $\frac{40}{78 s+1} e^{-3 s}$ \\
\hline
\end{tabular}

Both the partial correlations between the model residuals and the MVs and between the model residuals and the DV at each of the steady states are calculated. The results are plotted in Figs.3, 4 and 5. It can be seen from these figures that most of the partial correlations of the channels without model-plant mismatch is within the range of $[-0.15,0.15]$. The significant partial correlations between $e_{1}$ and MV2 in Fig. 3 and between $e_{2}$ and MV1, MV2 in Fig.5 correctly point out the mismatch channel listed in Table 1. The BFTs (Best Fitting) of the mismatch LPV model and the re-identified LPV model are compared in Table 2 . The comparison results show that the accuracy of the reidentified LPV model is much higher than that of the mismatch LPV model with respect to the best fittings of the two CVs.

$$
R_{\mathrm{BFT}}=100 * \max \left(1-\frac{\|y(k)-\hat{y}(k)\|_{2}}{\|y(k)-\bar{y}\|_{2}}, 0\right)
$$

where $y(k)$ is the process output, $\hat{y}(k)$ is the LPV model output and $\bar{y}$ is the average value of $y(k)$.
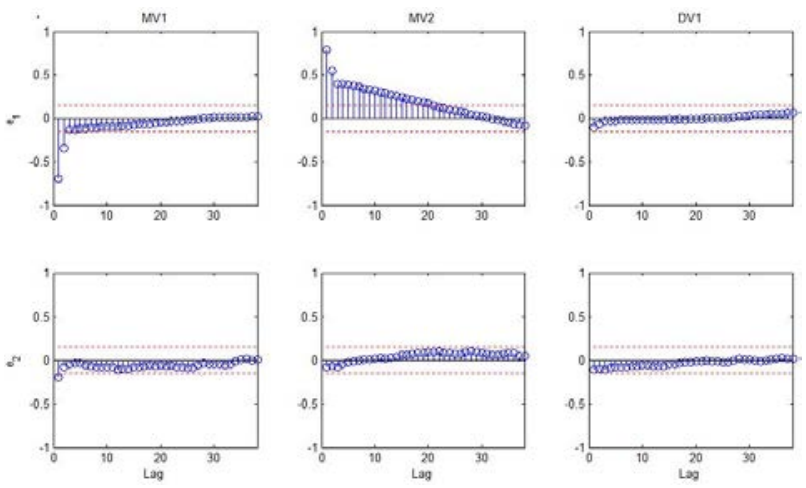

Fig. 3. Partial correlations plots at $w=1$
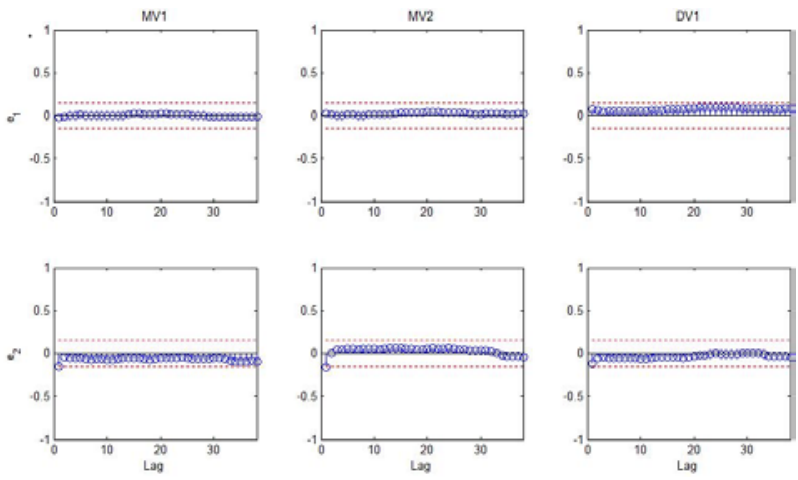

Fig. 4. Partial correlations plots at $w=2$
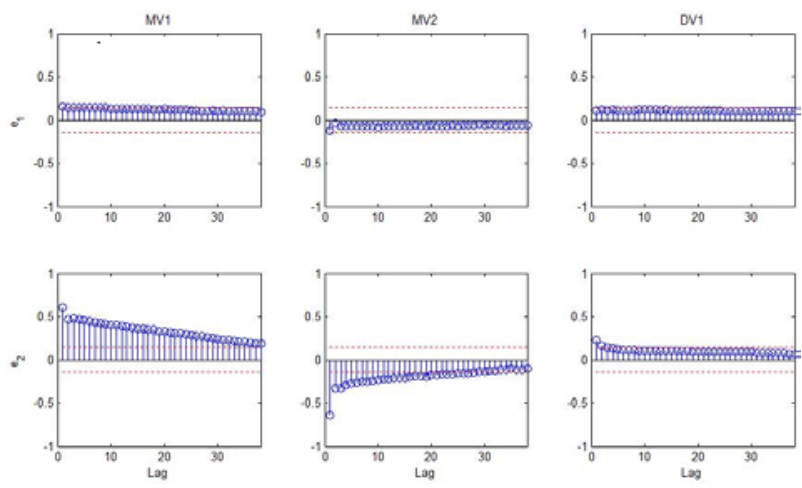

Fig. 5. Partial correlations plots at $w=3$

Table 2. BFTs of the mismatch LPV models

\begin{tabular}{|c|c|c|}
\hline & $\begin{array}{c}\text { BFTs of the } \\
\text { mismatch LPV model }\end{array}$ & $\begin{array}{c}\text { BFTs of the re- } \\
\text { identified LPV model }\end{array}$ \\
\hline CV1 & $75.5 \%$ & $92.4 \%$ \\
\hline CV2 & $69.8 \%$ & $91.6 \%$ \\
\hline
\end{tabular}

\section{Conclusions}

A methodology of model-plant mismatch detection for nonlinear processes based on LPV model is proposed in this work. The LPV model based on piecewise linear model interpolation is used to represent a nonlinear process, and then the model-plant mismatch of the local linear models at each of the operation points is diagnosed by partial correlation coefficient analysis. A 
new LPV model is obtained by estimating the weighting parameters after all the mismatch models are reidentified. The method proposed is suitable for most of the industrial processes which have relatively steady states in their operation trajectory. The main advantage of this method is that the mismatch of the local linear models at each operation point is diagnosed respectively and only the mismatch models need to be re-identified, which can reduce the cost of identifying the models of the entire process. The simulation results show that the partial correlation coefficient of the mismatch model is obviously larger than that of the accurate model, and the mismatch channel is correctly marked. Subsequent studies included the following aspects: (1) the simulation results show that the proposed method is sensitive to the mismatch of gain and time constant, but has a poor detection performance in time delay mismatch. The method should be improved so that the delay mismatch can be accurately detected. (2) The effectiveness of the proposed method in typical industrial processes should be verified. (3)The identification of the extent of modelplant mismatch and the impact of model-plant mismatch on controller performance should be studied. There is no need for re-identification when the mismatch is low or has little effect on the control performance.

\section{Acknowledgements}

This work is funded by the Fujian Natural Science Foundation of China(No. 2017J01509), the JK project of Education Department of Fujian(No. JK2015034) and the Opening Foundation of Advanced Process Control Laboratory for Light Industry of Jiangnan University (No. APCLI1601).

\section{References}

1. A.S. Badwe, R.D. Gudi, R.S. Patwardhan, S.L. Shah, S.C. Patwardhan, J. Process Control, 19, 1305-1313 (2009)

2. M. V. Loeff, A. Kuramoto, and C. Garcia. $X X$ Congresso Brasileiro de Automática,777-784,, 2014)

3. G. Ji, K.K. Zhang, Y.C. Zhu, J. Process Control, 22,635-642 (2012)

4. F. Yin, H. Wang, L. Xie, P. Wu, Z.H. Song,Comput. Elect. Eng, 40,2178-2192 (2014)

5. S. Yerramilli, A.K. Tangirala, IFAC-PapersOnLine, 1, 266-271(2016)

6. D. Ling, Y. Zheng, H.Zhang, W.D. Yang, B.Tao, J. Process Control, 57,66-79 (2017)

7. G. Chen, L. Xie, J. Zeng, J. Chu, Y. Gu,Ind.Eng.Chem. 52,1927-1938 (2013)

8. Y.C. Zhu, Z.H. Xu, Proc. of the 17th IFAC World Congress, Seoul, Korea (2008)

9. N. N. Nandola, S. Bhartiya, J. Process Control, 18, 131-148(2008)

10. J.Y. Huang, G.L. Ji, Y.C. Zhu, P.V.D. Bosch, J. Process Control,22,1198-1208 (2012) 